\title{
Bevacizumab Does Not Reduce the Lymphocele Rate in Advanced Ovarian Cancer After Complete Cytoreductive Surgery
}

\author{
MORGANE PERRIN $^{1}$, ENRICA BENTIVEGNA ${ }^{1}$, CLAIRE BONNEAU $^{1}$, \\ CATHERINE UZAN $^{1}$, ALEXANDRA LEARY ${ }^{2}$, PATRICIA PAUTIER ${ }^{2}$, \\ CATHERINE GENESTIE ${ }^{3}$, PHILIPPE MORICE ${ }^{1}$ and SÉBASTIEN GOUY ${ }^{1}$
}

\author{
Departments of ${ }^{1}$ Surgery, ${ }^{2}$ Oncology, and ${ }^{3}$ Pathology, Gustave Roussy Institute, Villejuif, France
}

\begin{abstract}
Background/Aim: We aimed to evaluate the impact of bevacizumab on the lymphocele rate in patients after complete cytoreductive surgery for advanced ovarian cancer. Patients and Methods: This retrospective study included patients with advanced ovarian cancer who had undergone complete cytoreductive surgery with pelvic and para-aortic lymphadenectomy at the Gustave Roussy Institute from 2005 to 2014. The introduction of bevacizumab was discussed in a multidisciplinary meeting. Results: During the study period, 247 patients were included; $24.6 \%$ of patients (61 patients) received adjuvant bevacizumab. The rate of symptomatic lymphocele was $34 \%$ (84 patients). In the lymphocele group, patients tended to receive adjuvant bevacizumab more often than did the control group $(32 \%$ and $21 \%$, respectively, $p=0.05)$. In multivariate analysis, bevacizumab was not significantly associated with the risk of symptomatic lymphocele (hazard ratio $(H R)=1.62,95 \%$ confidence interval $(C I)=0.87-3.01, p=0.12$ ). Conclusion: Adjuvant bevacizumab has no impact on the formation or duration of symptomatic lymphocele in patients after complete cytoreductive surgery for advanced ovarian cancer.
\end{abstract}

Ovarian cancer is the seventh most common malignancy among females worldwide, with an estimated incidence of about 239,000 new cases in the 2012 (1). More than $75 \%$ of cases are diagnosed at advanced stages due to the absence of symptoms in early stages and the prognosis is, therefore, poor (2). Treatment of advanced stages includes complete

Correspondence to: Sébastien Gouy, MD, Service de Chirurgie, Institut Gustave Roussy, 114 rue Edouard Vaillant, 94805 Villejuif Cedex, France. Tel: +33 142114439, Fax: +33 142115213, e-mail: sebastien.gouy@gustaveroussy.fr

Key Words: Lymphocele, ovarian cancer, bevacizumab, lymphadenectomy, surgery. cytoreductive surgery, which is a major prognostic factor (35), and adjuvant chemotherapy. Para-aortic and pelvic lymph node dissection is an integral part of surgical staging, and its benefit in terms of disease-free and overall survival (OS) have been demonstrated in retrospective studies $(6,7)$. In the only existing randomized trial, lymphadenectomy positively affected progression-free survival (8).

Complete cytoreductive surgery increases the risk of postoperative complications and lymphocele is one of the most frequent complications, but remains unstudied in the literature (9). Most lymphoceles are asymptomatic and resorb spontaneously (10). However, symptomatic lymphoceles can cause fever, abdominal pain, tenesmus, hydronephrosis, leg oedema and deep vein thrombosis. Symptomatic lymphoceles do not seem to have an impact on survival but do require needle aspiration, drainage or further surgery, which can prolong the hospital stay and delay the administration of adjuvant chemotherapy (11). The risk factors for lymphocele development are unclear. The extent of surgery and the number of lymph nodes removed seem to be risk factors in ovarian cancer (11). In gynaecological cancer, the extent of lymphadenectomy, presence of lymph node metastases, body mass index (BMI), lack of lymphatic vessel ligation and use of drainage have also been described as risk factors for lymphocele formation $(10,12-16)$. Yet, the lymphocele rate remains high because no clear management strategy has been established to reduce the risk of lymphocele formation (17) or to treat this complication.

The treatment strategy for advanced ovarian cancer was recently modified with the introduction of bevacizumab, a vascular endothelial growth factor (VEGF) inhibitor, in firstline treatment, combined with chemotherapy, and as maintenance therapy. Two trials, the International Collaboration on Ovarian Neoplasms (ICON) 7 (18) and Gynecologic Oncology Group (GOG) 02-18 (19) showed that adding bevacizumab to first-line adjuvant therapy prolonged recurrence-free survival by 2 or 4 months in 
patients with advanced epithelial ovarian cancer. The ICON 7 trial also showed that the benefits in terms of both recurrence-free survival and OS were greater among patients at high risk of progression (18). Since these trials, bevacizumab has been increasingly prescribed and the effect of this treatment has already been studied for malignant ascites in ovarian cancer and yielded a positive symptomatic benefit (20-23) but has never been studied on lymphoceles.

To our knowledge, this is the first study to investigate the impact of bevacizumab on the lymphocele rate in patients after complete cytoreductive surgery for advanced ovarian cancer.

\section{Patients and Methods}

Study population. This retrospective study included patients with advanced ovarian cancer, stages IIIB, IIIC, and IV according to the 1988 International Federation of Gynaecology and Obstetrics (FIGO) classification (24), who had received surgical treatment at our Institute from 2005 to 2014. All patients had undergone complete cytoreductive surgery with para-aortic and pelvic lymph node dissection. Patients were classified into a group with symptomatic lymphocele and a control group (without symptomatic lymphocele) and then into adjuvant bevacizumab group and control group (without adjuvant bevacizumab). Symptomatic lymphocele was defined as the presence of a lymphocele causing fever, abdominal pain, occlusion, and ureteral or vascular obstruction. Symptomatic lymphoceles systematically required needle aspiration, drainage under ultrasound or computed tomography guidance or further surgery. The following patient characteristics and surgical data were collected: age, BMI before surgery $\left(\mathrm{kg} / \mathrm{m}^{2}\right), 1988$ FIGO cancer stage, histological type (serous, mucinous, or other), type of surgery (standard, radical, or supra-radical), number of lymph nodes removed, lymph node involvement, and the length of hospital stay after surgery. Data on adjuvant therapy were also collected: neoadjuvant chemotherapy, adjuvant chemotherapy, the interval (days) between surgery and the initiation of chemotherapy, adjuvant bevacizumab, number of cycles, the interval (days) between surgery and initiation of bevacizumab, adverse events, and reasons for stopping bevacizumab early. In the group with symptomatic lymphocele, the number of needle aspiration or drainage events, and the interval (days) between the first and last such events were collected. In cases of repeated aspiration or drainage, a cytological analysis was performed.

Treatment and follow-up. Complete cytoreductive surgery was defined as no postoperative macroscopic residual tumour. Standard surgery comprised total abdominal hysterectomy, bilateral salpingooophorectomy, omentectomy, peritoneal biopsies in the pelvis and paracolic gutters, and systematic para-aortic and pelvic lymphadenectomy. Radical surgery included standard surgery with the Hudson procedure. In the latter, a rectosigmoidal resection along with resection of the reproductive organs and the pelvic peritoneum was accomplished using an en bloc retroperitoneal approach (25). Supra-radical surgery was performed in patients requiring resection of the diaphragm (full-thickness resection or partial or complete peritoneotomy), cholecystectomy, splenectomy, any bowel resection beyond removal of the sigmoid or an appendectomy, and resection of the bladder or ureter. Systematic lymphadenectomy included resection of pelvic and para-aortic lymph nodes. Pelvic lymphadenectomy included the removal of the common iliac, external and obturator nodal groups. Para-aortic lymphadenectomy was performed via a transperitoneal approach. The upper limit of the para-aortic dissection was the left renal vein. The pre-sacral chain, paracaval chain, and intercaval aortic chain were removed. The left para-aortic chain with the infra-mesenteric and supramesenteric groups was removed.

At the end of surgery, a pelvic drain with/without a retroperitoneal drain was left in place in the aortic area. In the case of diaphragmatic surgery or splenectomy, another drain was placed in the upper abdominal area. A low-pressure drain was always used. Withdrawal of drainage depended on the surgeons and outcomes. Drains were usually removed when the volume of fluid collected was less than 300-500 ml/day.

Patient follow-up included clinical examination and blood marker determination and the use of imaging techniques according to the findings every 4 months during the first 2 years, every 6 months up to 5 years, and then yearly thereafter.

Statistical analysis. The quantitative and qualitative variable results are expressed as medians and as frequencies and percentages, respectively. The statistical analysis was based on Student's $t$-test and the Mann-Whitney test for parametric and non-parametric continuous variables, respectively, and the chi-square test or Fisher's exact test, as appropriate, for categorical variables. $p$-Values of less than 0.05 were considered to denote significant differences. To assess the association between different variables and the occurrence of symptomatic lymphoceles, we used logistic regression analysis. Hazard ratios (HR) and the $95 \%$ confidence interval (CI) were calculated. Univariate analysis was followed by multivariate analysis. Variables in the univariate analysis had to reach a level of significance of 0.2 or had to be considered as a risk factor for lymphocele in the literature in order to be included in the multivariate model. The Kaplan-Meier method was used to estimate the survival distribution and survival rates were compared using the log-rank test. Data were managed with an Excel database (Microsoft, Redmond, WA, USA) and analysed using the R 2.15 software, available online.

\section{Results}

Two hundred and forty-seven patients had undergone complete cytoreductive surgery including a para-aortic and pelvic lymphadenectomy. Eighty-four patients (34\%) had a symptomatic lymphocele and 61 patients $(25 \%)$ had received adjuvant chemotherapy with bevacizumab. Table I shows the characteristics of patients with (lymphocele group) and without a lymphocele (control group). There was no difference between the two groups in terms of age, BMI, FIGO stage, histological subtype, type of surgery, number of para-aortic and pelvic nodes removed and chemotherapy characteristics (neoadjuvant and adjuvant, with or without bevacizumab). There was no difference between the two groups in terms of number of cycles of bevacizumab and the delay between complete cytoreductive surgery and the first cycle of bevacizumab. Conversely, the median hospital stay 
Table I. Characteristics of the two groups of patients included in this study.

\begin{tabular}{|c|c|c|c|}
\hline Characteristic & Lymphocele group $(\mathrm{n}=84)$ & Control group $(n=163)$ & $p$-Value \\
\hline Mean age (range), years & $53.4(23.3-74.0)$ & $54.4(25.2-70.3)$ & 0.51 \\
\hline Mean BMI (range), $\mathrm{kg} / \mathrm{m}^{2}$ & $25.5(17.4-40.4)$ & $24.5(16.5-36.1)$ & 0.14 \\
\hline Median hospital stay (range), days & $14(5-102)$ & $12(7-73)$ & 0.03 \\
\hline FIGO stage, $\mathrm{n}(\%)$ & & & 0.11 \\
\hline IIIB & 0 & $4(2 \%)$ & \\
\hline IIIC & $74(88 \%)$ & $149(92 \%)$ & \\
\hline IV & $10(12 \%)$ & $10(6 \%)$ & \\
\hline Histology, n (\%) & & & 0.28 \\
\hline Serous & $66(80 \%)$ & $119(73 \%)$ & \\
\hline Mucinous & $1(1 \%)$ & $8(5 \%)$ & \\
\hline Other & $16(19 \%)$ & $35(22 \%)$ & \\
\hline Unknown & 1 & 1 & \\
\hline \multicolumn{4}{|l|}{ Surgery, n (\%) } \\
\hline Standard & $14(17 \%)$ & $42(26 \%)$ & 0.10 \\
\hline Radical & $6(7 \%)$ & $19(11 \%)$ & 0.26 \\
\hline Supra-radical & $64(76 \%)$ & $102(63 \%)$ & 0.03 \\
\hline \multicolumn{4}{|l|}{ Median no. of lymph nodes removed (range) } \\
\hline Total & $50(17-95)$ & $42(6-123)$ & 0.05 \\
\hline Pelvic & $18(6-47)$ & $17(1-54)$ & 0.05 \\
\hline Para-aortic & $31(6-70)$ & $24(5-82)$ & 0.12 \\
\hline Patients with involved nodes, $\mathrm{n}(\%)$ & $62(74 \%)$ & $108(66 \%)$ & 0.22 \\
\hline Median no. of nodes involved (range) & $4(1-31)$ & $5(1-85)$ & 0.33 \\
\hline \multicolumn{4}{|l|}{ Chemotherapy } \\
\hline Neoadjuvant, n (\%) & $58(69 \%)$ & $103(63 \%)$ & 0.35 \\
\hline Adjuvant, n (\%) & $78(93 \%)$ & $147(93 \%)$ & 0.95 \\
\hline Bevacizumab, n (\%) & $27(32 \%)$ & $34(21 \%)$ & 0.05 \\
\hline Median no. of cycles of bevacizumab (range) & $16(2-36)$ & $16(1-36)$ & 0.69 \\
\hline Median delay between surgery and bevacizumab (range), days & $60(33-100)$ & $56(27-134)$ & 0.43 \\
\hline
\end{tabular}

BMI: Body mass index; FIGO: International Federation of Gynaecology and Obstetrics.

was significantly longer in the lymphocele group than in the control group, with 14 and 12 days, respectively ( $p=0.03)$.

Table II shows the results of multivariate analysis of the risk of lymphocele formation: no independent factor was associated with the risk of developing a symptomatic lymphocele postoperatively. Bevacizumab was not associated with the risk of developing a symptomatic lymphocele $(\mathrm{HR}=1.6295 \% \mathrm{CI}=0.87-3.01, p=0.12)$.

Table III shows the characteristics of the population with and without adjuvant bevacizumab. Both groups were comparable in terms of demographic characteristics (age and BMI), FIGO stage and type of surgery. Nevertheless, the group treated with adjuvant bevacizumab more frequently had serous histology $(88.5 \%$ vs. $71.5 \%)$ and less frequently mucinous histology (none vs. $4.8 \%$ ) than the group not treated with bevacizumab $(p=0.01)$. In addition, the group treated with adjuvant bevacizumab had more lymph nodes removed (49 vs. 43.5, $p=0.02$ ) than the group without bevacizumab.

Table IV shows that bevacizumab had no impact on radiological drainage. Eighty-four patients (34\%) developed symptomatic lymphoceles. Thirty-two patients (38\%) with a
Table II. Multivariate analysis of the association between variables and the occurrence of symptomatic lymphocele.

\begin{tabular}{llc}
\hline Covariate & HR $(95 \% \mathrm{CI})$ & $p$-Value \\
\hline Bevacizumab therapy & $1.62(0.87-3.01)$ & 0.12 \\
Supra-radical surgery & $1.78(0.97-3.38)$ & 0.06 \\
FIGO stage IV & $2.1(0.79-5.56)$ & 0.12 \\
$>40$ Lymph nodes removed & $1.58(0.90-2.83)$ & 0.11 \\
Lymph node involvement & $1.40(0.75-2.65)$ & 0.29 \\
Age $>60$ years & $1.11(0.61-2.01)$ & 0.72 \\
BMI $>25 \mathrm{~kg} / \mathrm{m}^{2}$ & $1.04(0.98-1.1)$ & 0.16 \\
\hline
\end{tabular}

HR: Hazard ratio, CI: confidence interval, BMI: body mass index, FIGO: International Federation of Gynaecology and Obstetrics.

symptomatic lymphocele had undergone only one needle aspiration (or drainage) and 12 at least two needle aspirations or drainage, with a median number of 3.5 (range=2-20). The median delay between surgery and the first needle aspiration was 34 days (range $=8-317$ days). 
Table III. Characteristics of the population treated with and without (Control) adjuvant bevacizumab.

\begin{tabular}{lcc}
\hline Characteristic & Bevacizumab (n=61) & Control group (n=186) \\
\hline Mean age (range), years & $57.9(23-70)$ & $54.1(24-74)$ \\
Mean BMI (range), $\mathrm{kg} / \mathrm{m}^{2}$ & $24.0(17-36)$ & $24.8(16-40)$ \\
FIGO stage, $\mathrm{n}(\%)$ & $1(2 \%)$ & $3(2 \%)$ \\
IIIB & $55(90 \%)$ & $168(90 \%)$ \\
IIIC & $5(8 \%)$ & $15(8 \%)$ \\
IV & $54(88 \%)$ & 0.83 \\
Histology, n (\%) & 0 & $131(71 \%)$ \\
Serous & $7(12 \%)$ & $9(5 \%)$ \\
Mucinous & & $44(24 \%)$ \\
Other & & 2 \\
Unknown & $9(14.8 \%)$ & 0.99 \\
Surgery, $n$ (\%) & $6(9.8 \%)$ & $19(25 \%)$ \\
Standard & $46(75.4)$ & $120(65 \%)$ \\
Radical & & 0.21 \\
Supra-radical & $49(19-123)$ & $44(6-108)$ \\
Median no. of lymph nodes removed (range) & $17(6-54)$ & $17(1-54)$ \\
Total & $30(7-82)$ & $26(5-73)$ \\
Pelvic & $44(72 \%)$ & $125(67 \%)$ \\
Para-aortic & $5(1-85)$ & $4(1-62)$ \\
Patients with involved nodes, n (\%) & $27(44 \%)$ & $57(31 \%)$ \\
Median no. of nodes involved (range) & & 0.02 \\
Symptomatic lymphocele & & 0.32 \\
\hline
\end{tabular}

BMI: Body mass index; FIGO: International Federation of Gynaecology and Obstetrics.

Table IV. Impact of adjuvant bevacizumab on drainage of symptomatic lymphocele.

\begin{tabular}{|c|c|c|c|}
\hline Characteristic & Bevacizumab & Control group & $p$-Value \\
\hline No. of patients with only one needle aspiration/drainage & $10(37 \%)$ & $22(38 \%)$ & 0.89 \\
\hline Median no. of needle aspirations/drainage if $>1$, (range) & $8(2-16)$ & $4(2-20)$ & 0.54 \\
\hline Median delay between first and last needle aspiration/drainage (range), days & $33(4-357)$ & $74(5-400)$ & 0.33 \\
\hline
\end{tabular}

In the bevacizumab-treated and the control groups, the median follow-up times were 27.1 (range=13.7-75.3) months and 40.8 (range $=1.3-107.4$ ) months, respectively.

\section{Discussion}

The risk factors for development of lymphocele are unclear. The extent of surgery and number of lymph nodes removed seem to be risk factors in ovarian cancer (11). In gynaecological cancer, the extent of the lymphadenectomy, the presence of lymph node metastases, the BMI, the lack of lymphatic vessel ligation and the use of drainage have also been described as risk factors for lymphocele formation $(10$, 12-16).

Our results show that bevacizumab did not reduce the lymphocele rate after complete cytoreductive surgery in advanced ovarian cancer. To our knowledge, our series is the largest in the literature (247 patients having undergone complete cytoreductive surgery and a complete para-aortic and pelvic lymphadenectomy) to investigate the lymphocele rate in the bevacizumab era.

Lymphocele occurs very frequently after complete paraaortic and pelvic lymphadenectomy but has been poorly studied. The only study which raised the question of the frequency of symptomatic lymphocele after cytoreductive surgery in advanced ovarian cancer found a rate of $28 \%$ (11). It broached the issue of the quality of life of patients who had undergone extensive and complete surgery. In our study, $34 \%$ of patients had developed a symptomatic lymphocele which required at least one needle aspiration (or drainage) and $14 \%$ had undergone more than one needle aspiration. Regarding the hospital stay, we found it was significantly longer for patients with symptomatic lymphocele but only by approximately 2 days, which confirms the result of the study 
reported by Gauthier et al. (11). We did not find a significant delay in the introduction of adjuvant treatment unlike that previous study, which is very important because adjuvant therapy is a decisive step in the treatment of patients with advanced ovarian cancer.

These results do not challenge the indication for systematic lymphadenectomy for this subgroup of patients if we consider the quality of life and survival associated with lymphocele. In the literature, several studies showed the benefit of lymphadenectomy in optimal surgery (6-8). More precisely, Du Bois et al. found that total lymphadenectomy afforded an OS benefit for patients with advanced ovarian cancer and cytoreductive surgery with no residual tumour $(\mathrm{HR}=0.74,95 \% \mathrm{CI}=0.59-0.94, p=0.0123)(6)$. This signifies that lymphadenectomy is an important prognostic factor for such patients as well as there being no residual tumour at the end of surgery. Panici et al. reported that systematic lymphadenectomy alone afforded a benefit for progressionfree survival but in women who had undergone optimal surgery (residual tumour $\leq 1 \mathrm{~cm}$ in $60 \%$ of cases and no residual tumour in $37 \%$ of cases). Our population had undergone complete cytoreductive surgery at a major referral centre applying guidelines and with surgeons highly experienced in extensive surgery.

In our study, we found no risk factor for symptomatic lymphocele formation. In the literature, Gautier et al. found that undergoing supra-radical surgery was an independent risk factor for symptomatic lymphocele formation in a multivariate analysis in ovarian cancer, as well as removal of more than 40 lymph nodes in the univariate analysis. Despite a larger study population, we did not identify supra-radical surgery and the number of lymph nodes removed $(>40)$ as significant risk factors in our multivariate analysis $(\mathrm{HR}=1.78$, 95\% $\mathrm{CI}=0.97-3.38, p=0.06$ and $\mathrm{HR}=1.58,95 \% \mathrm{CI}=0.90-2.83$, $p=0.11$ respectively). Other factors such as a high BMI, the extent of the lymphadenectomy and the presence of lymph node metastasis have been described to promote the formation of lymphocele in gynaecological cancer $(10,12$, 16). None of these factors were proven to be independent risk factors in our study, that confirms previous results.

In the literature, therapy with intravenous or intraperitoneal bevacizumab seems to be beneficial in patients with malignant ascites in ovarian cancer and decreases the number of needle aspirations required (20-23). The hypothesis put forward for this benefit was the expression of VEGF in the fluid. Kobold et al. showed that VEGF was overexpressed in malignant ascites in comparison with non-malignant ascites (21). We did not verify VEGF expression in lymphoceles but it seems obvious that it would be low after complete cytoreductive surgery. Unsurprisingly, bevacizumab did not reduce the lymphocele rate $(\mathrm{HR}=1.62,95 \% \mathrm{CI}=0.87-3.01$, $p=0.12$ in the multivariate analysis) nor did it lower the number of needle aspirations (or drainage) required.
One potential bias of our retrospective study was the number of lymph nodes removed, which was significantly higher in the bevacizumab-treated group (49 versus 44 in the group without bevacizumab). Several studies have demonstrated the benefit of total lymphadenectomy in terms of survival for patients with advanced ovarian cancer who had undergone optimal surgery (6-8) and the number of lymph nodes removed was a prognostic factor with a cut-off point of 10 lymph nodes in the study conducted by Rouzier et al. (7). In our study, all the patients had undergone complete cytoreductive surgery with no residual tumour and pelvic and para-aortic lymphadenectomy, and only two of them had fewer than 10 lymph nodes removed in the entire study population. Finally, our study showed that the number of lymph nodes removed was not a risk factor for lymphocele development in the multivariate analysis.

A second potential bias was the cancer histological types: there were more patients with serous tumours and fewer with mucinous histology in the bevacizumab-treated group than in the group without bevacizumab. Du Bois et al. found that a mucinous histological type was a strong prognostic factor for survival versus a serous histological type in debulked advanced ovarian cancer (3). In our study, the number of mucinous tumours was very low in the two groups $(1 \%$ and $5 \%$ in the groups treated without and with bevacizumab, respectively). These very low numbers reduced the impact of this potential bias.

\section{Conclusion}

Adjuvant bevacizumab has no impact on the formation or the duration of symptomatic lymphocele.

\section{References}

1 Ferlay J, Soerjomataram I, Dikshit R, Eser S, Mathers C, Rebelo M, Parkin DM, Forman D and Bray F: Cancer incidence and mortality worldwide: Sources, methods and major patterns in GLOBOCAN 2012. Int J Cancer 136: E359-386, 2015.

2 Siegel R, Naishadham D and Jemal A: Cancer statistics, 2013. CA Cancer J Clin 63: 11-30, 2013.

3 du Bois A, Reuss A, Pujade-Lauraine E, Harter P, Ray-Coquard I and Pfisterer J: Role of surgical outcome as prognostic factor in advanced epithelial ovarian cancer: a combined exploratory analysis of 3 prospectively randomized phase 3 multicenter trials: by the Arbeitsgemeinschaft Gynaekologische Onkologie Studiengruppe Ovarialkarzinom (AGO-OVAR) and the Groupe d'Investigateurs Nationaux Pour les Etudes des Cancers de l'Ovaire (GINECO). Cancer 115: 1234-1244, 2009.

4 Eisenkop SM, Spirtos NM, Friedman RL, Lin W-CM, Pisani AL and Perticucci S: Relative influences of tumor volume before surgery and the cytoreductive outcome on survival for patients with advanced ovarian cancer: a prospective study. Gynecol Oncol 90: 390-396, 2003.

5 Eisenkop SM, Spirtos NM and Lin W-CM: "Optimal" cytoreduction for advanced epithelial ovarian cancer: a commentary. Gynecol Oncol 103: 329-335, 2006. 
6 du Bois A, Reuss A, Harter P, Pujade-Lauraine E, Ray-Coquard I, Pfisterer J, Arbeitsgemeinschaft Gynaekologische Onkologie Studiengruppe Ovarialkarzinom and Groupe d'Investigateurs Nationaux pour l'Etude des Cancers Ovariens: Potential role of lymphadenectomy in advanced ovarian cancer: a combined exploratory analysis of three prospectively randomized phase III multicenter trials. J Clin Oncol 28: 1733-1739, 2010.

7 Rouzier R, Bergzoll C, Brun J-L, Dubernard G, Selle F, Uzan S, Pomel $\mathrm{C}$ and Daraï $\mathrm{E}$ : The role of lymph node resection in ovarian cancer: analysis of the Surveillance, Epidemiology, and End Results (SEER) database. Int J Obstet Gynaecol 117: 14511458, 2010.

8 Benedetti Panici P, Basile S, Maneschi F, Alberto Lissoni A, Signorelli M, Scambia G, Angioli R, Tateo S, Mangili G, Katsaros D, Garozzo G, Campagnutta E, Donadello N, Greggi S, Melpignano M, Raspagliesi F, Ragni N, Cormio G, Grassi R, Franchi M, Giannarelli D, Fossati R, Torri V, Amoroso M, Crocè $\mathrm{C}$ and Mangioni C: Systematic pelvic lymphadenectomy vs. no lymphadenectomy in early-stage endometrial carcinoma: randomized clinical trial. J Natl Cancer Inst 100: 1707-1716, 2008.

9 Chéreau E, Ballester M, Lesieur B, Selle F, Coutant C, Rouzier $\mathrm{R}$ and Daraï E: Complications of radical surgery for advanced ovarian cancer. Gynécologie Obstétrique Fertil 39: 21-27, 2011 (in French).

10 Kim HY, Kim JW, Kim SH, Kim YT and Kim JH: An analysis of the risk factors and management of lymphocele after pelvic lymphadenectomy in patients with gynecologic malignancies. Cancer Res Treat 36: 377-383, 2004.

11 Gauthier T, Uzan C, Lefeuvre D, Kane A, Canlorbe G, Deschamps F, Lhomme C, Pautier P, Morice P and Gouy S: Lymphocele and ovarian cancer: risk factors and impact on survival. Oncologist 17: 1198-1203, 2012.

12 Ghezzi F, Uccella S, Cromi A, Bogani G, Robba C, Serati M and Bolis P: Lymphoceles, lymphorrhea, and lymphedema after laparoscopic and open endometrial cancer staging. Ann Surg Oncol 19: 259-267, 2012.

13 Gallotta V, Fanfani F, Rossitto C, Vizzielli G, Testa A, Scambia $\mathrm{G}$ and Fagotti A: A randomized study comparing the use of the Ligaclip with bipolar energy to prevent lymphocele during laparoscopic pelvic lymphadenectomy for gynecologic cancer. Am J Obstet Gynecol 203: 483.e1-6, 2010.

14 Benedetti-Panici P, Maneschi F, Cutillo G, D'Andrea G, di Palumbo VS, Conte M, Scambia G and Mancuso S: A randomized study comparing retroperitoneal drainage with no drainage after lymphadenectomy in gynecologic malignancies. Gynecol Oncol 65: 478-482, 1997.
15 Morice P, Lassau N, Pautier P, Haie-Meder C, Lhomme C and Castaigne D: Retroperitoneal drainage after complete Para-aortic lymphadenectomy for gynecologic cancer: a randomized trial. Obstet Gynecol 97: 243-247, 2001.

16 Petru E, Tamussino K, Lahousen M, Winter R, Pickel H and Haas J: Pelvic and para-aortic lymphocysts after radical surgery because of cervical and ovarian cancer. Am J Obstet Gynecol 161: 937-941, 1989.

17 Gauthier T, Gouy S, Uzan C, Kane A and Morice P: Prevention of lymphoceles and gynaecologic cancers. Gynécologie Obstétrique Fertil 39: 698-703, 2011 (in French).

18 Perren TJ, Swart AM, Pfisterer J, Ledermann JA, PujadeLauraine E, Kristensen G, Carey MS, Beale P, Cervantes A, Kurzeder C, du Bois A, Sehouli J, Kimmig R, Stähle A, Collinson F, Essapen S, Gourley C, Lortholary A, Selle F, Mirza MR, Leminen A, Plante M, Stark D, Qian W, Parmar MKB, Oza AM and ICON7 Investigators: A phase 3 trial of bevacizumab in ovarian cancer. N Engl J Med 365: 2484-2496, 2011.

19 Burger RA, Brady MF, Bookman MA, Fleming GF, Monk BJ, Huang H, Mannel RS, Homesley HD, Fowler J, Greer BE, Boente M, Birrer MJ, Liang SX and Gynecologic Oncology Group: Incorporation of bevacizumab in the primary treatment of ovarian cancer. N Engl J Med 365: 2473-2483, 2011.

20 Hamilton CA, Maxwell GL, Chernofsky MR, Bernstein SA, Farley JH and Rose GS: Intraperitoneal bevacizumab for the palliation of malignant ascites in refractory ovarian cancer. Gynecol Oncol 111: 530-532, 2008.

21 Kobold S, Hegewisch-Becker S, Oechsle K, Jordan K, Bokemeyer $\mathrm{C}$ and Atanackovic D: Intraperitoneal VEGF inhibition using bevacizumab: a potential approach for the symptomatic treatment of malignant ascites? Oncologist 14: 1242-1251, 2009.

22 Eskander RN and Tewari KS: Emerging treatment options for management of malignant ascites in patients with ovarian cancer. Int J Womens Health 4: 395-404, 2012.

23 Smolle E, Taucher V and Haybaeck J: Malignant ascites in ovarian cancer and the role of targeted therapeutics. Anticancer Res 34: 1553-1561, 2014.

24 FIGO Cancer Committee Staging Announcement: FIGO staging of ovarian cancer. Gynecol Oncol: 383-385, 1986.

25 Hudson $\mathrm{CN}$ : A radical operation for fixed ovarian tumours. J Obstet Gynaecol Br Commonw 75: 1155-1160, 1968.

Received October 31, 2017

Revised February 2, 2018

Accepted February 5, 2018 